\title{
Conjugate Effects of Thermal and Mass Diffusion on Natural Convection Flow from a Heated Sphere with Internal Heat Generation or Absorption
}

\author{
Sadia Masud and Nepal Chandra Roy* \\ Department of Mathematics, Dhaka University, Dhaka 1000, Bangladesh
}

(Received: 30 April 2018; Accepted: 5 December 2018)

\begin{abstract}
We examine the conjugate effects of thermal and mass diffusion on the unsteady natural convection flow from a heated sphere. The mathematical model of the problem is made into a system of nonsimilar partial differential equations introducing appropriate transformations. We solve this system employing the implicit finite difference technique. Numerical solutions have been elucidated with the skin friction as well as heat and mass transfer for varying Prandtl number, Schmidt number, combined buoyancy parameter, and heat generation or absorption parameter.
\end{abstract}

Keywords: Natural convection, Heat generation, Mass Transfer.

\section{Introduction}

There are many engineering and natural phenomena in which fluid flow and heat transfer processes are ubiquitous. The combined buoyancy force resulting from the thermal and mass diffusions affects numerous processes occurring in industry and in the environment. Moreover, several scientific fields, namely, geology, biology, astrophysics, oceanography, chemical processes and crystal growth techniques are influenced by variation of density because of the temperature and chemical species gradients and material or phase constitution. Besides, the buoyancy driven flow induced by diffusion of thermal and chemical species is also a concern in the underground containment storage, augmentation cooling of micro processes, petroleum geology as well as nuclear engineering.

Due to the extensive practical importance, this topic has received much attention from the researchers. Siddiqa et al. ${ }^{1}$ investigated heat transfer from a vertical wavy surface resulting from the natural convection. Roy and Hossain ${ }^{2}$ studied the consequence of thermal radiation on the fluctuating free convective boundary layer along a vertical plate. Cao and Baker ${ }^{3}$ observed the non-continuum effects of interfacial discontinuity on natural convection boundary layer flow. But all the accounts mentioned above only concentrated on the thermal diffusion neglecting chemical species diffusion processes. The condition is considered to be general when the flow is induced by temperature differences and is combined with the mass diffusion of the same order of magnitude. So neglecting one over the other could produce incorrect results. Understanding this background the combined effect of thermal as well as mass diffusion was considered by a number of researchers. Pera and Gebhart ${ }^{4}$ conducted an analysis to reveal the flow behavior under the conjugate influence of thermal and chemical species diffusion. Siddiqa et al. ${ }^{5}$ examined the natural convective heat and mass transfer behavior along a wavy surface with thermal radiation. $\mathrm{Pal}^{6}$ investigated the characteristics of stagnation point flow over a vertical stretching sheet of an incompressible fluid considering buoyancy forces and thermal radiation. Gebhart and Pera studied the combined buoyancy effect owing to the thermal and chemical species diffusion. Hossain ${ }^{8}$ examined the heat and mass transfer behavior in mixed convection flow along a permeable vertical plate. Later, Hussain et al. ${ }^{9}$ considered the similar problem for natural convective flow along a flat plate. Abdallah and Zeghmati ${ }^{10}$ have sought to work on the combined effect of thermal and buoyancy force along the wavy surface. However, the above-mentioned works did not realize the effect of time variation on the flow conditions. Besides of all these literature, none of them involved spherical geometry even though this geometry is relevant to numerous industrial and natural phenomena. Nguyen and Paik ${ }^{11}$ analyzed the fluctuating behavior of the transport phenomena including the effects density changes of the involved fluid. Potter and Riley ${ }^{12}$ examined the characteristics of the boundary layer adjacent the sphere surface as well as the eruption of the fluid from boundary layer into the plume region. But the prior literature did not include both thermal diffusion and heat generation or absorption at the same time. Later, Prasad et al. ${ }^{13}$ studied the non-Darcy magnetohydro-dynamics natural convective heat transfer flow from a circular cylinder. Recently, Roy and Hossain ${ }^{14}$ have observed the effects of thermal radiation as well as heat generation or absorption for unsteady mixed convection flow. These two projects included thermal diffusion as well as heat generation or absorption simultaneously. So taking inspiration from Prasad et al. ${ }^{13}$ and Roy and Hossain ${ }^{14}$ we have considered the effects of heat source-sink with thermal and mass diffusion.

Due to the significance of spherical geometry, the present work aims to analyze the unsteady natural convection flow from a sphere taking into account the thermal and mass diffusion and heat source-sink. The effects of pertinent parameters on the nature of fluid flow are examined and presented graphically.

\section{Mathematical Formulations}

A two-dimensional, steady, laminar, natural convection flow is considered over a sphere which is assumed to be suddenly heated. We suppose that the surface temperature and species concentration experience an angular 
displacement $\bar{x}$ from the lower pole. The coordinates and the physical domain of the problem are elucidated in Fig. 1.

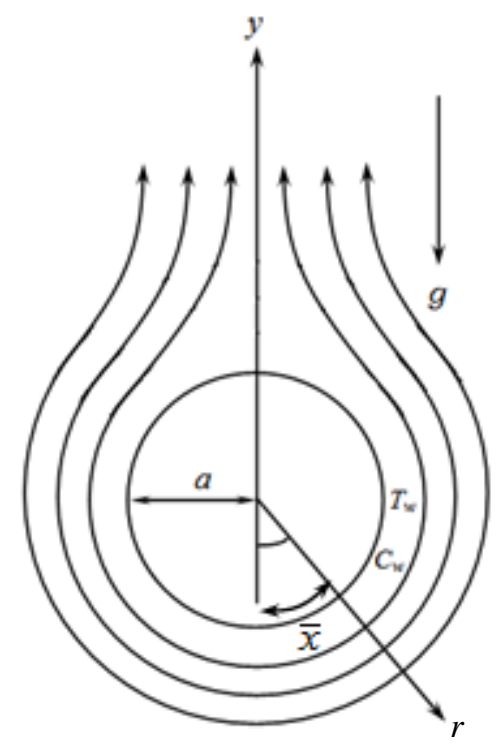

Fig. 1. Physical model of the problem

Here $r$ is the measure of distance normal to the sphere, $(u$, $v)$ denotes component of velocity along the $(x, r)$ directions, $a$ is the radius of the sphere. Under the Boussinesq approximations, the governing equations of the problem, following equations ${ }^{14,15}$, are

$\frac{\partial}{\partial x}(r u)+\frac{\partial}{\partial r}(r v)=0$

$\frac{\partial u}{\partial t}+u \frac{\partial u}{\partial x}+v \frac{\partial u}{\partial r}=\frac{v}{r} \frac{\partial}{\partial r}\left(r \frac{\partial u}{\partial r}\right)$

$+g\left\{\beta_{T}\left(T-T_{e}\right)+\beta_{C}\left(C-C_{e}\right)\right\} \sin \left(\frac{x}{a}\right)$

$\frac{\partial T}{\partial t}+u \frac{\partial T}{\partial x}+v \frac{\partial T}{\partial r}=\frac{\alpha}{r} \frac{\partial}{\partial r}\left(r \frac{\partial T}{\partial r}\right)+\frac{Q}{\rho c_{p}}\left(T-T_{e}\right)$

$\frac{\partial C}{\partial t}+u \frac{\partial C}{\partial x}+v \frac{\partial C}{\partial r}=\frac{D}{\rho r} \frac{\partial}{\partial r}\left(r \frac{\partial C}{\partial r}\right)$

subject to the boundary conditions are

$u=v=0, T=T_{e}, C=C_{e}$ at $r>0, t=0$

$\left.\begin{array}{l}u=v=0, T=T_{w}, C \rightarrow C_{w} \text { at } r=a \\ u=0, T \rightarrow T_{e}, C \rightarrow C_{e} \text { as } r \rightarrow \infty .\end{array}\right\}, t>0$.

Here $t$ is the time, $T$ the temperature, $C$ the concentration of the species and $v, c_{p}, g, \alpha, D$ and $\rho$ denotes the kinematic viscosity, specific heat capacity, acceleration due to gravity, thermal diffusivity, mass diffusivity and density respectively. The volumetric expansion coefficient of temperature and concentration are represented by $\beta_{T}$ and $\beta_{C}$ respectively. In addition, when $Q>0$ it refers to the heat generation but when $Q<0$ it indicates heat absorption and the coefficient $Q\left(T-T_{e}\right)$ introduced in equation (3) represents the generation or absorption of heat per unit volume. The subscript $e$ denotes the ambient conditions or references state. At the beginning, the surface temperature of the sphere is raised to a temperature $T_{w}>T_{e}$ and then maintained at $T_{w}$. Accordingly, there generates fluid motion over the sphere due to the temperature gradients.

The following dimensionless variables are introduced,

$$
\begin{aligned}
& x=\frac{x}{a}, R=\frac{r}{a}, y=\frac{r-a}{a} G r_{L}^{1 / 4}, \zeta=\frac{U_{0} t}{a}, U=\frac{u}{U_{0}}, \\
& V=\frac{v}{U_{0}} G r_{L}^{1 / 4}, \theta=\frac{T-T_{e}}{T_{w}-T_{e}}, \phi=\frac{C-C_{e}}{C_{w}-C_{e}} .
\end{aligned}
$$

where $U_{0}=v G r_{L}^{1 / 2} / a$ is the characteristic velocity and $r=\sin x$.

Therefore, the governing equations (1)-(4) take the form

$\frac{\partial(U \sin x)}{\partial x}+\frac{\partial(V \sin x)}{\partial y}=0$

$\frac{\partial U}{\partial \zeta}+U \frac{\partial U}{\partial x}+V \frac{\partial U}{\partial y}=\frac{\partial^{2} U}{\partial y^{2}}$

$+\{(1-w) \theta+w \phi\} \sin x$

$\frac{\partial \theta}{\partial \zeta}+U \frac{\partial \theta}{\partial x}+V \frac{\partial \theta}{\partial y}=\frac{1}{\operatorname{Pr}} \frac{\partial^{2} \theta}{\partial y^{2}}+\gamma \theta$

$\frac{\partial \phi}{\partial \zeta}+U \frac{\partial \phi}{\partial x}+V \frac{\partial \phi}{\partial y}=\frac{1}{S c} \frac{\partial^{2} \phi}{\partial y^{2}}$.

with boundary conditions

$U=V=0, \quad \theta=0, \quad \phi=0$ for $y>0$ and $\zeta=0$

$\left.\begin{array}{l}U=V=0, \quad \theta=1, \phi=1 \text { at } y=0 \\ U=V=0, \quad \theta=0, \phi=0 \text { as } y \rightarrow \infty .\end{array}\right\}, \zeta>0$.

Here,

$G r_{L}=G r_{T}+G r_{C}$

$=\frac{g \beta_{T}\left(T_{w}-T_{e}\right) a^{3}}{v^{2}}+\frac{g \beta_{c}\left(C_{w}-C_{e}\right) a^{3}}{v^{2}}$

is the global Grashof number where $G r_{T}$ and $G r_{C}$ are the Grashof numbers for thermal diffusion and mass diffusion, respectively. Also $w=G r_{C} /\left(G r_{T}+G r_{C}\right)$ is the combined buoyancy parameter. It is noted that when $w=0$ there is no species diffusion and when $w \rightarrow 1$ there is no thermal diffusion. In equations (8)-(11), the pertinent parameters are Prandtl number, $\operatorname{Pr}=v / \alpha$, heat generation or absorption parameter, $\gamma=Q a^{2} /\left(\rho v c_{p} G r_{L}^{1 / 2}\right)$ and Schmidt number, $S c$ $=v / D$.

The following transformations are used in order to solve the present problem numerically, 


$$
\begin{aligned}
& \eta=(1+\operatorname{Pr})^{1 / 4}\left(1-e^{-\tau}\right)^{-1 / 2} y, \xi=x, \\
& \psi=(1+\operatorname{Pr})^{-3 / 4}\left(1-e^{-\tau}\right)^{3 / 2} \sin ^{2} x F(\tau, \xi, \eta), \\
& \tau=\ln (1+\zeta), \theta=\theta(\tau, \xi, \eta), \phi=\phi(\tau, \xi, \eta) .
\end{aligned}
$$

Here $\eta$ is the similarity variable, $\xi$ is the distance, $\theta$ denotes the dimensionless temperature and $\phi$ is the dimensionless concentration. Now we define the stream function $\psi$ by,

$$
\frac{\partial \psi}{\partial y}=U \sin x \text { and }-\frac{\partial \psi}{\partial x}=V \sin x .
$$

which satisfies the equation (1).

Applying equations (15) and (16) on equations (9)-(12) the momentum, energy and concentration equations becomes,

$$
\begin{aligned}
& (1+\operatorname{Pr}) F^{\prime \prime \prime}+(1+\operatorname{Pr})^{1 / 2} e^{-2 \tau}\left\{\frac{1}{2} \eta F^{\prime \prime}-F^{\prime}\right\} \\
& +(1+\operatorname{Pr})\{(1-w) \theta+w \phi\} \\
& +\cos \xi\left(1-e^{-\tau}\right)^{2}\left(2 F F^{\prime \prime}-F^{\prime 2}\right) \\
& =(1+\operatorname{Pr})^{1 / 2}\left(1-e^{-\tau}\right) e^{-\tau} \frac{\partial F^{\prime}}{\partial \tau} \\
& +\left(1-e^{-\tau}\right)^{2} \sin \xi\left(F^{\prime} \frac{\partial F^{\prime}}{\partial \xi}-F^{\prime \prime} \frac{\partial F}{\partial \xi}\right) \\
& \frac{(1+\operatorname{Pr})}{\operatorname{Pr}} \theta^{\prime \prime}+\frac{1}{2}(1+\operatorname{Pr})^{1 / 2} e^{-2 \tau} \eta \theta^{\prime} \\
& +2 \cos \xi\left(1-e^{-\tau}\right)^{2} F \theta^{\prime}+(1+\operatorname{Pr})^{1 / 2} \gamma\left(1-e^{-\tau}\right) \theta \\
& =(1+\operatorname{Pr})^{1 / 2}\left(1-e^{-\tau}\right) e^{-\tau} \frac{\partial \theta}{\partial \tau} \\
& +\left(1-e^{-\tau}\right)^{2} \sin \xi\left(F^{\prime} \frac{\partial \theta}{\partial \xi}-\theta^{\prime} \frac{\partial F}{\partial \xi}\right) \\
& \frac{(1+\operatorname{Pr})}{S c} \phi^{\prime \prime}+\frac{1}{2}(1+\operatorname{Pr})^{1 / 2} e^{-2 \tau} \eta \phi^{\prime} \\
& +2 \cos \xi\left(1-e^{-\tau}\right)^{2} F \phi^{\prime} \\
& =(1+\operatorname{Pr})^{1 / 2}\left(1-e^{-\tau}\right) e^{-\tau} \frac{\partial \phi}{\partial \tau} \\
& +\left(1-e^{-\tau}\right)^{2} \sin \xi\left(F^{\prime} \frac{\partial \phi}{\partial \xi}-\phi^{\prime} \frac{\partial F}{\partial \xi}\right) \text {. }
\end{aligned}
$$

Finally the boundary conditions becomes,

$$
\left.\begin{array}{l}
F^{\prime}=F=0, \quad \theta=0, \phi=0 \text { for } \eta>0 \text { and } \tau=0 \\
F^{\prime}=F=0, \quad \theta=1, \phi=1 \text { at } \eta=0 \\
F^{\prime}=F=0, \quad \theta=0, \phi=0 \text { as } \eta \rightarrow \infty .
\end{array}\right\}, \tau>0 .
$$

Here the prime indicates the differentiation with respect to the similarity variable $\eta$. At this stage, equations (16)-(18) subjected to the boundary conditions (19) can be solved numerically utilizing implicit finite difference technique.

\section{Numerical Method}

The momentum, energy, and concentration equations (16)(18) subjected to the boundary condition (19) are solved numerically employing finite difference technique. From scientific and engineering point of view, the skin friction and the rate of heat and mass transfer are important. Therefore, measurable physical quantities, like skin friction, $\tau_{x}$, rate of heat transfer, $q_{x}$, and rate of mass transfer $m_{x}$ are used to express the flow behavior. The mathematical expressions of these quantities take the underlining forms,

$$
\begin{aligned}
& \bar{\tau}_{x}=\mu\left(\frac{\partial \bar{u}}{\partial \bar{r}}\right)_{\bar{r}=a}, \bar{q}_{x}=-\alpha\left(\frac{\partial T}{\partial \bar{r}}\right)_{\bar{r}=a}, \\
& \bar{m}_{x}=-D\left(\frac{\partial C}{\partial \bar{r}}\right)_{\bar{r}=a} .
\end{aligned}
$$

After substituting equation (7) and using equations (14)(15) on equation (21) the parameters become

$$
\begin{aligned}
\tau_{x} & =\left(1-e^{-\tau}\right)^{-1 / 2}(1+\operatorname{Pr})^{1 / 4} \sin \xi f^{\prime \prime}(\tau, 0, \xi), \\
q_{x} & =\left(1-e^{-\tau}\right)^{-1 / 2}(1+\operatorname{Pr})^{1 / 4} \theta^{\prime}(\tau, 0, \xi), \\
m_{x} & =\left(1-e^{-\tau}\right)^{-1 / 2}(1+\operatorname{Pr})^{1 / 4} \phi^{\prime}(\tau, 0, \xi),
\end{aligned}
$$

where

$$
\begin{aligned}
& \tau_{x}=\frac{a^{2} \bar{\tau}_{x}}{\mu \nu G r_{L}^{3 / 4}}, q_{x}=\frac{a \bar{q}_{x}}{\alpha\left(T_{w}-T_{e}\right) G r_{L}^{1 / 4}}, \\
& m_{x}=\frac{a \bar{m}_{x}}{\alpha\left(C_{w}-C_{e}\right) G r_{L}^{1 / 4}} .
\end{aligned}
$$

Utilizing the values of $f^{\prime \prime}, \theta^{\prime}$ and $\phi^{\prime}$ one can easily estimate the skin friction coefficient, rate of heat transfer and rate of mass transfer from equations (22).

\section{Results and Discussions}

We have studied a steady natural convection flow of incompressible fluid over a spherical object. The governing equations (17) - (19) subjected to the boundary conditions (20) are solved by finite difference method. Results are described with the variations of the Prandtl number, $\mathrm{Pr}$, the Schmidt number, Sc, combined buoyancy parameter, $w$, heat generation/absorption parameter, $\gamma$. For different non similarity variable, $\xi$ the flow is also observed.

Numerical data are represented with the skin friction, heat transfer and mass transfer Fig. 2(a)-(c) respectively for $\xi=0.5,1.0,1.5,2.0,2.5$ while $\operatorname{Pr}=0.72, S c=0.22, \gamma=0.5$ and $w=0.5$. Fig. 2 shows that for higher values of $\xi$, the skin friction is augmented but the heat and mass transfer rates become weaker. As the value of $\xi$ increases, the buoyancy force accelerates the flow but decelerates the heat flux and mass transfer. It should be mentioned that the skin friction and the rate of heat and mass transfer become steady state at $\tau=8.0$. In this regard, the results presented in the following figures are for steady state condition. 

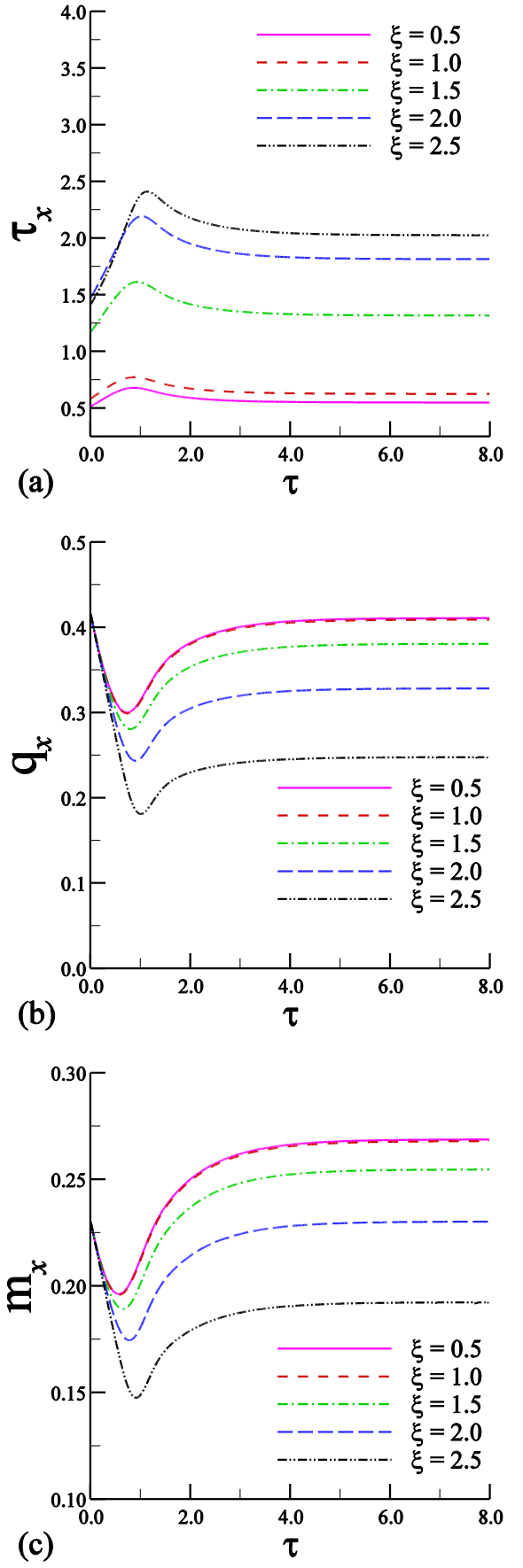

Fig. 2. Effect of variation of $\xi$ on (a) skin friction, (b) heat transfer, and (c) mass transfer.

The variations of the skin friction, heat transfer and mass transfer with the Prandtl number, $\mathrm{Pr}$, are demonstrated in Figs. 3(a)-(c). It is evident from Fig. 3(a) that the skin friction first increases with $\xi<2.0$ and then it decreases. It is due to the change of curvature of the sphere. For higher values of the Prandtl number, Pr, the skin friction and heat transfer are found to increase while the rate of mass transfer decreases. The reason is attributed from the definition of $\operatorname{Pr}=v / \alpha$ that the Prandtl number increases either for higher values of kinematics viscosity or for lower values of thermal diffusivity.
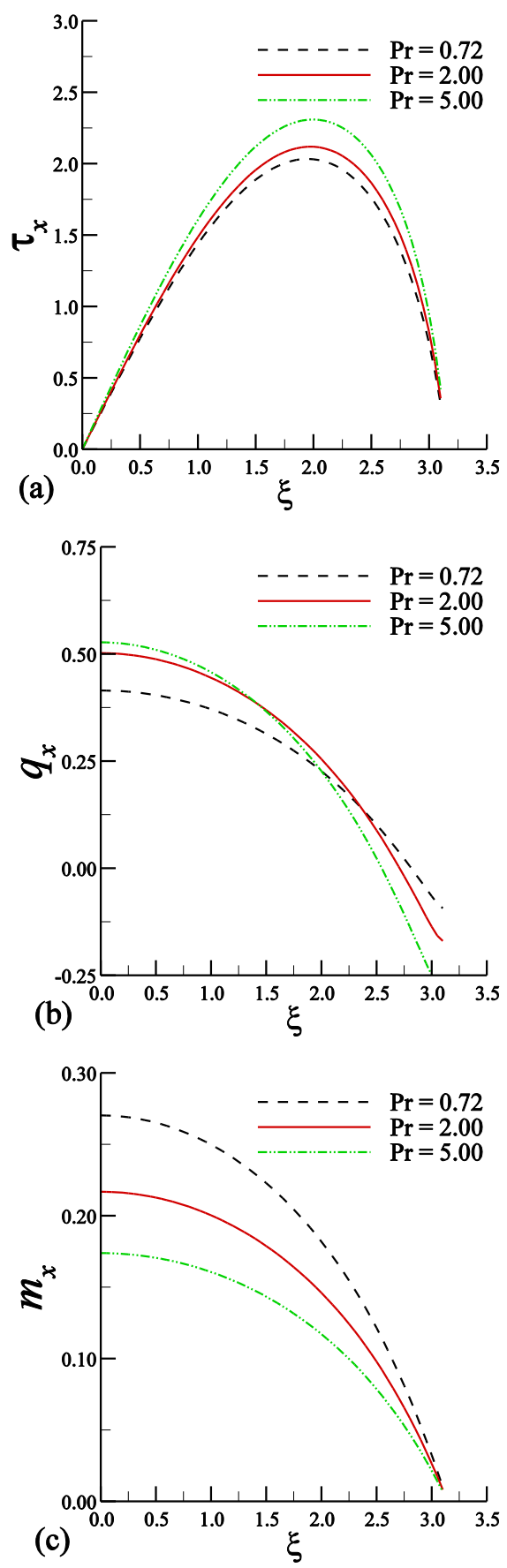

Fig. 3. Effect of variation of $\operatorname{Pr}$ on (a) skin friction, (b) heat transfer, and (c) mass transfer.

The effects of different Schmidt number, $S c$, on the skin friction and the rate of heat and mass transfer are shown in Figs. 4(a)-(c) respectively. With an increase of $S c$ the skin friction and heat transfer decrease while mass transfer rate is increased. It is seen that the value of Schmidt number, $S c$ $=v / D$, represents the relative importance of kinematic viscosity to mass diffusivity. So, the Schmidt number is higher for smaller values of mass diffusivity or larger values of kinematic viscosity. For this reason, the mass transfer is stronger for larger values of Schmidt number. 

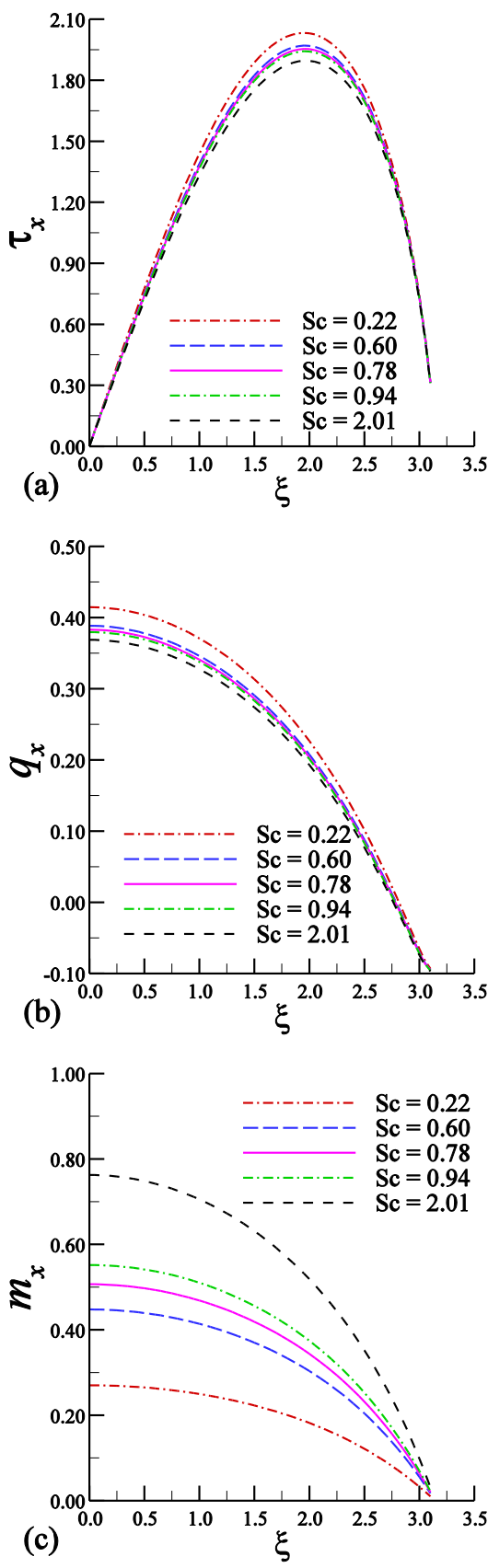

Fig. 4. Effect of variation of $S c$ on (a) skin friction, (b) heat transfer, and (c) mass transfer.

Figures 5(a)-(c) demonstrate the influence of the variations of dimensionless heat generation or absorption parameter on the skin friction and the rate of heat and mass transfer. It is observed from figures that when the intensity of a heat source $(\gamma>0)$ becomes stronger, the skin friction and the rate of mass transfer grow while reverse characteristics is observed for heat transfer. On the contrary, the skin friction and rate of mass transfer increase owning to the increase of the heat absorption $(\gamma<0)$ parameter while the rate of heat transfer decreases. It is obvious that when the heat absorption increases or the heat generation becomes lower, the temperature gradients on the sphere surface must be higher and consequently the heat transfer increases.
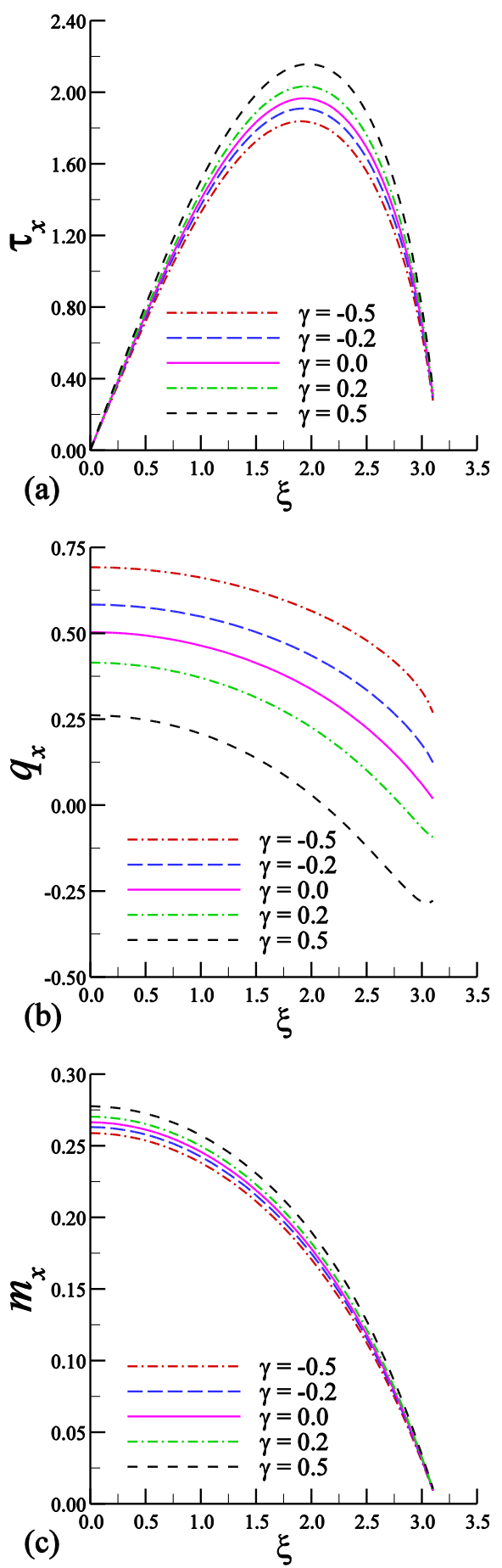

Fig. 5. Effect of variation of $\gamma$ on (a) skin friction, (b) heat transfer, and (c) mass transfer.

The influence of the combined buoyancy parameter, $w$, on the skin friction, heat transfer and mass transfer is depicted in Figs. 6(a)-(c) respectively. It is observed from the figures that an increase in the value of $w$ causes an augmentation of the skin friction, rate of heat and mass transfer rate. It is because that the conjugate effect of thermal and mass diffusion enhances the drag on the surface, mass diffusion and heat transfer. 

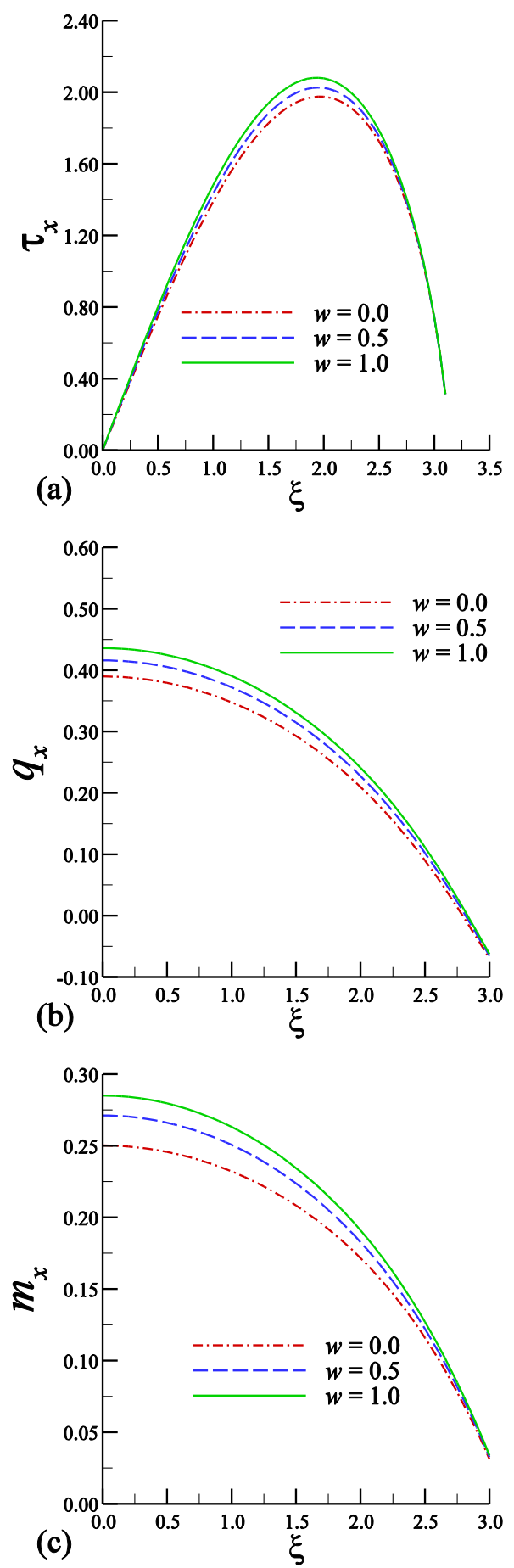

Fig. 6. Effect of variation of $w$ on (a) skin friction, (b) heat transfer, and (c) mass transfer.

\section{Conclusions}

The present work aims to study the unsteady natural convection flow from a sphere with the conjugate effects of thermal and mass diffusion, and heat source or sink variations. The governing equations are made dimensionless using appropriate transformations. We solve these equations numerically using implicit finite difference technique. Then the influence of the physical parameters on the nature of the flow is observed and presented graphically. It is revealed that the higher Prandtl number reduces the mass transfer but augments the skin friction and heat transfer. With an increment of the Schmidt number the skin friction and heat transfer decrease whereas the rate of mass transfer is significantly increased. It is seen that the skin friction, rate of heat transfer and rate of mass transfer considerably increase due to the increase of the combined buoyancy parameter. For larger internal heat generation, the skin friction and mass transfer are increased but the rate of heat transfer decreases. However, the reverse situation is observed with the change of heat absorption parameter.

\section{References}

1. Siddiqa, S., M. Mahfooz and M.A. Hossain, 2018. Transient analysis of heat transfer by natural convection along a vertical wavy surface, Int. J. Nonlinear Sci. Num. Simul., 18, $175-195$.

2. Roy, N. C. and M.A. Hossain, 2010. The effect of conduction-radiation on the oscillating natural convection boundary layer flow of viscous incompressible fluid along a vertical plate, Proc. Inst. Mech. Eng.: Part C: J. Mech. Eng. Sci. 224, 1959-1972.

3. Cao, K. and J. Baker, 2015. Non-continuum effects on natural convection-radiation boundary layer flow from a heated vertical plate, Int. J. Heat Mass Transf., 90, 26-33.

4. Pera, L. and B. Gebhart, 1972. Natural convection flows adjacent to horizontal surfaces resulting from the combined buoyancy effects of thermal and mass diffusion, Int. J. Heat Mass Transf., 15, 269-278.

5. Siddiqa, S., M.N. Abrar, M.A. Hossain, and R.S.R. Gorla, 2017. Double diffusive natural convection flow over a wavy surface situated in a non-absorbing medium, Int. J. Heat Mass Transf., 109, 200-208.

6. Pal, D., 2009. Heat and mass transfer in stagnation-point flow towards a stretching surface in the presence of buoyancy force and thermal radiation, Meccanica, 44(2), 145-158.

7. Gebhart, B. and L. Pera, 1971, The nature of vertical natural convection flow resulting from the combined buoyancy effects of thermal and mass diffusion, Int. J. Heat Mass Transf., 14, 2025-2050.

8. Hossain, M. A., 1992. Effect of transpiration on combined heat and mass transfer in mixed convection along a vertical plate, Int. J. Energy Res., 16, 761-769.

9. Hussain, S., M.A. Hossain and M. Wilson, 2000. Natural convection flow from a vertical permeable flat plate with variable surface temperature and species concentration, Engineering Computations, 17, 789-812.

10. Abdallah, M. S. and B. Zeghmati, 2014. Heat and mass transfer due to natural convection along a wavy vertical plate with opposing thermal and solutal buoyancy effects, Fluid Dyn. Mater. Proc., 10(2), 261-277.

11. Nguyen, H. D. and S. Paik, 1994. Unsteady mixed convection from a sphere in water-saturated porous media with variable surface temperature/heat flux, Int. J. Heat Mass Transf., 37, 1783-1793. 
12. Potter, J. and N. Riley, 1980. Free convection from a heated sphere at large Grashof number, J. Fluid Mech., 100,769783.

13. Prasad, V. R., A.S. Rao and O.A. B’eg, 2014. Computational analysis of viscous dissipation and joule-heating effects on non-Darcy MHD natural convection flow from a horizontal cylinder in porous media with internal heat generation, Theor. Appl. Mech., 41, 37-70.

14. Roy, N. C. and M.A. Hossain, 2014. Numerical solution of a steady natural convection flow from a vertical plate with the combined effects of streamwise temperature and species concentration variations, Heat Mass Transf., 46, 509-522.

15. Roy, N. C., M.A. Hossain and R.S.R. Gorla, 2015. Unsteady free convection from a heated sphere in the presence of internal heat generation or absorption, Int. J. Therm. Sci., 98, 237-244. 
